\title{
Delay-Decomposition Stability Approach of Nonlinear Neutral Systems with Mixed Time-Varying Delays
}

\author{
Ilyes MAZHOUD \\ University of Tuins El Manar, National \\ Engineering School of Tunis, \\ Laboratory of research in Automatic \\ Control, BP 37, Belvédère, 1002 \\ Tunis, Tunisia
}

\author{
Issam AMRI \\ University of Tuins El Manar, National \\ Engineering School of Tunis, \\ Laboratory of research in Automatic \\ Control, BP 37, Belvédère, 1002 \\ Tunis, Tunisia
}

\author{
Dhaou SOUDANI \\ University of Tuins El Manar, National \\ Engineering School of Tunis, \\ Laboratory of research in Automatic \\ Control, BP 37, Belvédère, 1002 \\ Tunis, Tunisia
}

\begin{abstract}
This paper deals with the asymptotic stability of neutral systems with mixed time-varying delays and nonlinear perturbations. Based on the Lyapunov-Krasovskii functional including the triple integral terms and free weighting matrices approach, a novel delay-decomposition stability criterion is obtained. The main idea of the proposed method is to divide each delay interval into two equal segments. Then, the LyapunovKrasovskii functional is used to split the bounds of integral terms of each subinterval. In order to reduce the stability criterion conservatism, delay-dependent sufficient conditions are performed in terms of Linear Matrix Inequalities (LMIs) technique. Finally, numerical simulations are given to show the effectiveness of the proposed stability approach.
\end{abstract}

Keywords-Neutral systems; Lyapunov-Krasovskii approach; asymptotic stability; mixed time-varying delays; nonlinear perturbations; Linear Matrix Inequalities (LMIs)

\section{INTRODUCTION}

Neutral time-delay appears in many fields of sciences and engineering, including neural networks, industrial, economy, chemical processes and population models. In fact, the presence of time-delay causes the instability, the oscillation, and performances' degradation of dynamical systems. Neutral systems are a part of a specific class of infinite dimensions. Their stability study can be a complex issue. Recently, the stability problem of neutral systems has been the subject of considerable research [1-23]. Thus, several approaches of delay-dependent stability criteria have been developed for this problem.

The stability criteria of neutral systems with mixed timevarying delays can be classified into two concepts. Firstly, the delay-dependent stability which is based on the size of timedelay and it gives the upper bound of delay in the formulation. Secondly, the delay-independent stability class doesn't include any information about the size of the time-delay. Indeed, the delay-dependent is often less conservative than the delayindependent.

In order to reduce the conservatism, many researchers studied the nonlinear neutral systems stability with mixed time-varying delays such as in [1] where authors consider the delay-dependent robust stability of uncertain neutral systems with mixed time-varying delays. In [2], I. Amri et al. have been studied a delay-dependent exponential stability condition for nonlinear neutral systems with mixed delays. They employ a delay-decomposition approach and the known free weighting matrices method.In [3], novel delay-decomposition condition of neutral systems with time-varying delays is proposed and new stability results were derived. In [4], the authors have been presented a new asymptotic stability results for nonlinear neutral system with mixed delays by using the delay-dividing approach. In [5], the exponential stability of neutral delay differential systems with nonlinear uncertainties is used. The problem of the delay-dependent robust stability criteria for neutral systems with mixed time-varying delays and nonlinear perturbations has been studied in [6]. In [7], new less conservative robust stability criteria of neutral systems with mixed time-varying delays and nonlinear perturbations are derived by using the delay method.

In this paper, the problem of asymptotical delaydecomposition stability for nonlinear neutral systems with mixed time-varying delays is investigated. By using a new augmented Lyapunov-Krasovskii functional including the triple integral terms for interval time-varying delays as well as the free-weighting matrices technique and Jensen integral inequality, new sufficient delay-dependent stability conditions have been proposed and expressed in terms of LMIs. These stability conditions can be easily solved by various convex optimization algorithms.

The remainder of this paper is organized as follows. In Section 2, the stability problem of nonlinear neutral systems is described. Some related preliminaries are also given. The main result of this paper is presented in Section 3. Numerical examples are carried out in Section 4 in order to illustrate the proposed results. Section 5 concludes this paper.

\section{PROBLEM DESCRIPTION AND PRELIMINARIES}

This paper considers the nonlinear neutral systems with mixed time-varying delays of Equation (1): 


$$
\left\{\begin{aligned}
& \dot{x}(t)-A_{2} \dot{x}(t-\tau(t))= A x(t)+A_{1} x(t-h(t)) \\
&+f_{0}(t, x(t)) \\
&+f_{1}(t, x(t-h(t))) \\
&+f_{2}(t, \dot{x}(t-\tau(t))) \\
& x(t)=\varphi(t), \dot{x}(t)=\phi(t), \quad \forall t \in\left[-\max \left\{\tau_{M}, h_{M}\right\}, 0\right]
\end{aligned}\right.
$$

where $x(t) \in \square^{n}$ is the state vector $A, A_{1}, A_{2} \in \square^{n \times n}$ are constant matrices with appropriate dimensions. $\tau(t), h(t)$ are neutral and discrete time-varying delays satisfying the following equations:

$$
\begin{array}{ll}
0<h_{m} \leq h(t) \leq h_{M}, & \dot{h}(t) \leq \mu<1, \\
0<\tau_{m} \leq \tau(t) \leq \tau_{M}, & \dot{\tau}(t) \leq \eta<1,
\end{array}
$$

The initial conditions functions $\varphi(t), \phi(t)$ are continuously differentiable on $\left[-\max \left\{\tau_{M}, h_{M}\right\}, 0\right]$. The functions $f_{0}(t, x(t))$,

$$
f_{1}(t, x(t-h(t))) \text { and } f_{2}(t, \dot{x}(t-\tau(t))) \text { are unknown nonlinear }
$$
uncertainties satisfying $f_{0}(t, 0)=0, f_{1}(t, 0)=0, f_{2}(t, 0)=0$ and

$$
\left\{\begin{array}{l}
\left\|f_{0}(t, x(t))\right\| \leq \beta_{0}\|x(t)\|, \\
\left\|f_{1}(t, x(t-h(t)))\right\| \leq \beta_{1}\|x(t-h(t))\|, \\
\left\|f_{2}(t, \dot{x}(t-\tau(t)))\right\| \leq \beta_{2}\|\dot{x}(t-\tau(t))\|,
\end{array}\right.
$$

where $\beta_{0} \geq 0, \beta_{1} \geq 0, \beta_{2} \geq 0$ are given constants.

Constraint (4) can be rewritten as follows:

$$
\left\{\begin{array}{l}
f_{0}^{T}(t, x(t)) f_{0}(t, x(t)) \leq \beta_{0}^{2} x^{T}(t) x(t) \\
f_{1}^{T}(t, x(t-h(t))) f_{1}(t, x(t-h(t))) \leq \beta_{1}^{2} x^{T}(t-h(t)) x(t-h(t)) \\
f_{2}^{T}(t, \dot{x}(t-\tau(t))) f_{2}(t, \dot{x}(t-\tau(t))) \leq \beta_{2}^{2} \dot{x}^{T}(t-\tau(t)) \dot{x}(t-\tau(t))
\end{array}\right.
$$

For simplicity, note that:

$$
\left.f_{0}:=f_{0}(t, x(t))\right), f_{1}:=f_{1}(t, x(t-h(t))), f_{2}:=f_{2}(t, \dot{x}(t-\tau(t)))
$$

Moreover, for dividing the each interval time-varying delay into two equal subintervals $\left[h_{m}, \alpha_{1} h_{M}\right] \operatorname{or}\left[\alpha_{1} h_{M}, h_{M}\right]$ and $\left[\tau_{m}, \alpha_{2} \tau_{M}\right]$ or $\left[\alpha_{2} \tau_{M}, \tau_{M}\right]$, two different cases for time-varying delays have been presented.

Case I: $h(t), \tau(t)$ are differentiable functions, satisfying for all $t \geq 0$ :

$$
\begin{gathered}
h_{m} \leq h(t) \leq h_{M} \quad \text { and } \dot{h}(t) \leq \mu<1, \\
\tau_{m} \leq \tau(t) \leq \tau_{M} \quad \text { and } \dot{\tau}(t) \leq \eta<1 .
\end{gathered}
$$

Case II: $h(t)$ is not differentiable or the upper bound of the derivative of $h(t)$ and $\tau(t)$ is a differentiable function, and $h(t), \tau(t)$ satisfying:

$$
\begin{aligned}
& h_{m} \leq h(t) \leq h_{M}, \\
& \tau_{m} \leq \tau(t) \leq \tau_{M}, \quad \dot{\tau}(t) \leq \eta<1 .
\end{aligned}
$$

where $h_{m}, h_{M}, \tau_{m}, \tau_{M}, d$ and ${ }^{\mu}$ are positive scalars.
This paper is devoted to investigate the delay-dependent stability analysis of time-varying delays system (1) satisfying (2) and (3) equations and under nonlinear perturbations inequalities (4) and (5). It aims to formulate a less conservative stability technique to estimate the upper bound for the delay interval. Before deriving the proposed stability criteria, the following lemmas are needed.

\section{Lemma 1. [8]}

For any constant matrix $R \in \square^{n \times n}, R=R^{T}>0$, a scalar function $h:=h(t)>0$, and a vector valued function $\dot{x}:[-h, 0] \rightarrow \square^{n}$ such that the following integrations are well defined, then:

$$
\begin{aligned}
& -h \int_{t-h}^{t} \dot{x}^{T}(s) R \dot{x}(s) d s \leq \psi_{1}^{T}(t)\left[\begin{array}{cc}
-R & R \\
R & -R
\end{array}\right] \psi_{1}(t) \\
& -\frac{h^{2}}{2} \int_{-h t+\theta}^{0} \int_{t}^{t} \dot{x}^{T}(s) R \dot{x}(s) d s d \theta \leq \psi_{2}^{T}(t)\left[\begin{array}{cc}
-R & R \\
R & -R
\end{array}\right] \psi_{2}(t)
\end{aligned}
$$

where $\psi_{1}^{T}(t)=\left[x^{T}(t) x^{T}(t-h)\right]$ and $\psi_{2}^{T}(t)=\left[h x^{T}(t) \int_{t-h}^{t} x^{T}(s) d s\right]$.

Lemma 2. [9]: The following matrix inequality

$$
\left(\begin{array}{cc}
Q(x) & S(x) \\
S^{T}(x) & R(x)
\end{array}\right)<0,
$$

where $Q(x)=Q^{T}(x), \quad R(x)=R^{T}(x)$ and $\quad S(x)$ depend on affine on $x$, is equivalent to $R(x)<0, Q(x)<0$ and $Q(x)-S(x) R^{-1}(x) S^{T}(x)<0$.

Lemma 3. [10]: For any scalar $\tau(t) \geq 0$ and any constant matrix $R \in \square^{n \times n}, R=R^{T}>0$, the following inequality holds:

$$
\begin{aligned}
&-\int_{t-\alpha_{1} h_{M}}^{t-h(t)} \dot{x}^{T}(s) R \dot{x}(s) d s \leq\left(\alpha_{1} h_{M}-h(t)\right) \xi^{T}(t) F R^{-1} F^{T} \xi(t) \\
&+2 \xi^{T}(t) F\left[x(t-h(t))-x\left(t-\alpha_{1} h_{M}\right)\right]
\end{aligned}
$$

where

$\xi^{T}(t)=\left[\begin{array}{l}x^{T}(t) x^{T}\left(t-h_{m}\right) x^{T}(t-h(t)) x^{T}\left(t-\alpha_{1} h_{M}\right) x^{T}\left(t-\tau_{m}\right) x^{T}(t-\tau(t)) x^{T}\left(t-\alpha_{2} \tau_{M}\right) \\ \dot{x}^{T}(t) \dot{x}^{T}(t-\tau(t))\left(\int_{t-\alpha_{2} \tau_{M}}^{t} x(s) d s\right)^{T}\left(\int_{t-\alpha_{1} h_{M}}^{t-h_{m}} x(s) d s\right)^{T}\left(\int_{t-\alpha_{2} \tau_{M}}^{t-\tau_{m}} x(s) d s\right)^{T} f_{0}^{T} f_{1}^{T} f_{2}^{T}\end{array}\right]$

and $F$ is free-weighting matrix with appropriate dimensions.

\section{MAIN RESULTS}

In order to obtain some less conservative conditions, new delay-decomposition method for nonlinear neutral system (1) is developed. The first delay-interval $\left[h_{m}, h_{M}\right]$ is divided into two segments $\left[h_{m}, \alpha_{1} h_{M}\right]$ and $\left[\alpha_{1} h_{M}, h_{M}\right]$. The second delayinterval $\left[\tau_{m}, \tau_{M}\right]$ is decomposed into two subintervals $\left[\tau_{m}, \alpha_{2} \tau_{M}\right]$ and $\left[\alpha_{2} \tau_{M}, \tau_{M}\right]$. The following theorem presents 
new stability criteria for interval time-varying delay system (1).

Theorem1. In Case I, if $h_{m} \leq h(t) \leq \alpha_{1} h_{M}\left(0<\alpha_{1}<1\right)$ and $\tau_{m} \leq \tau(t) \leq \alpha_{2} \tau_{M} \quad\left(0<\alpha_{2}<1\right)$, for given positive scalars $h_{m}, h_{M}, \tau_{m}, \tau_{M}, \eta, \mu, \beta_{0}, \beta_{1}$ and $\beta_{2}$, the system (1) with uncertainty (5) and mixed time-varying delays satisfying(2) and (3) is asymptotically stable if there exist symmetric positive definite $n \times n$ matrices $P, Q_{i}(i=1, . ., 7), R_{j}(j=1, . ., 7)$, for any free matrix variables $T_{a}, Y_{a}, W_{a}, N_{a}, X_{a}, F_{a}(a=1,2)$ and scalars $\varepsilon_{i} \geq \mathrm{O}(\boldsymbol{i}=\mathrm{O}, 1,2)$ such that the following symmetric LMI holds:

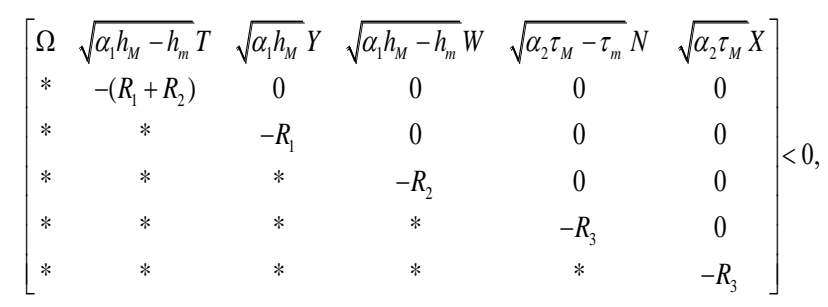

where $\Omega=\left(\Omega_{i, j}\right)_{15 \times 15}$ and:

$\Omega_{1,1}=Q_{1}+Q_{2}+Q_{3}+Q_{4}+Q_{6}+Q_{7}+Y_{1}+Y_{1}^{T}+X_{1}+X_{1}^{T}$

$-2 R_{5}-2 R_{6}-2 R_{7}+F_{1} A+A^{T} F_{1}^{T}+\varepsilon_{0} \beta_{0}^{2} \mathrm{I}$

$\Omega_{1,3}=-Y_{1}+Y_{2}^{T}+F_{1} A_{1}$

$\Omega_{1,6}=-X_{1}+X_{2}^{T}$

$\Omega_{1,8}=P-F_{1}+A^{T} F_{2}^{T}$

$\Omega_{1,9}=F_{1} A_{2}$

$\Omega_{1,10}=\frac{2}{\alpha_{2} \tau_{M}} R_{5}$

$\Omega_{1,11}=\frac{2}{\left(\alpha_{1} h_{M}+h_{m}\right)} R_{6}$

$\Omega_{1,12}=\frac{2}{\left(\alpha_{2} \tau_{M}+\tau_{m}\right)} R_{7}$

$\Omega_{1,13}=\Omega_{1,14}=\Omega_{1,15}=F_{1}$

$\Omega_{2,2}=-Q_{3}+W_{1}+W_{1}^{T}$

$\Omega_{2,3}=-W_{1}+W_{2}^{T}$

$\Omega_{3,3}=-(1-\mu) Q_{2}+T_{1}+T_{1}^{T}-Y_{2}-Y_{2}^{T}-W_{2}-W_{2}^{T}+\varepsilon_{1} \beta_{1}^{2} \mathrm{I}$

$\Omega_{3,4}=-T_{1}+T_{2}^{T}$

$\Omega_{3,8}=A_{1}^{T} F_{2}^{T}$

$\Omega_{4,4}=-Q_{1}-T_{2}-T_{2}^{T}$

$\Omega_{5,5}=-R_{4}-Q_{7}$

$\Omega_{5,7}=R_{4}$

$$
\begin{aligned}
& \Omega_{6,6}=-(1-\eta) Q_{4}+N_{1}+N_{1}^{T}-X_{2}-X_{2}^{T} \\
& \Omega_{7,7}=-R_{4}-Q_{6}-N_{2}-N_{2}^{T} \\
& \Omega_{8,8}=M-F_{2}-F_{2}^{T} \\
& \Omega_{8,9}=F_{2} A_{2} \\
& \Omega_{8,13}=\Omega_{8,14}=\Omega_{8,15}=F_{2} \\
& \Omega_{9,9}=-(1-\eta) Q_{5}+\varepsilon_{2} \beta_{2}^{2} \mathrm{I} \\
& \Omega_{10,10}=-\frac{2}{\alpha_{2}^{2} \tau_{M}^{2}} R_{5} \\
& \Omega_{11,11}=-\frac{2}{\left(\alpha_{1}^{2} h_{M}^{2}-h_{m}^{2}\right)} R_{6} \\
& \Omega_{12,12}=-\frac{2}{\left(\alpha_{2}^{2} \tau_{M}^{2}-\tau_{m}^{2}\right)} R_{7} \\
& \Omega_{13,13}=-\varepsilon_{0} \mathrm{I} \\
& \Omega_{14,14}=-\varepsilon_{1} \mathrm{I} \\
& \Omega_{15,15}=-\varepsilon_{2} \mathrm{I} \\
& M=Q_{5}+\alpha_{1} h_{M} R_{1}+\left(\alpha_{1} h_{M}-h_{m}\right) R_{2}+\alpha_{2} \tau_{M} R_{3}+\left(\alpha_{2} \tau_{M}-\tau_{m}\right)^{2} R_{4} \\
& +\frac{\alpha_{2}^{2} \tau_{M}^{2}}{2} R_{5}+\frac{\left(\alpha_{1}^{2} h_{M}^{2}-h_{m}^{2}\right)}{2} R_{6}+\frac{\left(\alpha_{2}^{2} \tau_{M}^{2}-\tau_{m}^{2}\right)}{2} R_{7}
\end{aligned}
$$

Proof. Choose a new augmented Lyapunov-Krasovskii functional as:

$$
V(t)=V_{1}(t)+V_{2}(t)+V_{3}(t)+V_{4}(t)
$$

where

$$
\begin{aligned}
V_{1}(t) & =x^{T}(t) P x(t), \\
V_{2}(t) & =\int_{t-\alpha_{1} h_{M}}^{t} x^{T}(s) Q_{1} x(s) d s+\int_{t-h(t)}^{t} x^{T}(s) Q_{2} x(s) d s+\int_{t-h_{m}}^{t} x^{T}(s) Q_{3} x(s) d s \\
& +\int_{t-\tau(t)}^{t} x^{T}(s) Q_{4} x(s) d s+\int_{t-\tau(t)}^{t} \dot{x}^{T}(s) Q_{5} \dot{x}(s) d s+\int_{t-\alpha_{2} \tau_{M}}^{t} x^{T}(s) Q_{6} x(s) d s \\
& +\int_{t-\tau_{m}}^{t} x^{T}(s) Q_{7} x(s) d s, \\
V_{3}(t) & =\int_{-\alpha_{1} h_{M}}^{0} \int_{t+\theta}^{t} \dot{x}^{T}(s) R_{1} \dot{x}(s) d s d \theta+\int_{-\alpha_{1} h_{M}}^{-h_{h_{m}}} \int_{t+\theta}^{t} \dot{x}^{T}(s) R_{2} \dot{x}(s) d s d \theta \\
& +\int_{-\alpha_{2} \tau_{M}}^{0} \int_{t+\theta}^{t} \dot{x}^{T}(s) R_{3} \dot{x}(s) d s d \theta+\left(\alpha_{2} \tau_{M}-\tau_{m}\right) \int_{-\alpha_{2} \tau_{M}}^{-\tau_{m}} \int_{t+\theta}^{t} \dot{x}^{T}(s) R_{4} \dot{x}(s) d s d \theta, \\
V_{4}(t) & =\int_{-\alpha_{2} \tau_{M}}^{0} \int_{\theta}^{0} \int_{t+\lambda}^{t} \dot{x}^{T}(s) R_{5} \dot{x}(s) d s d \lambda d \theta+\int_{-\alpha_{1} h_{M}}^{-h_{m}} \int_{\theta t+\lambda}^{t} \int_{t}^{T}(s) R_{6} \dot{x}(s) d s d \lambda d \theta \\
& +\int_{-\alpha_{m} \tau_{M}}^{0} \int_{\theta}^{t} \int_{t+\lambda}^{t} \dot{x}^{T}(s) R_{7} \dot{x}(s) d s d \lambda d \theta . \\
& \text { with }
\end{aligned}
$$




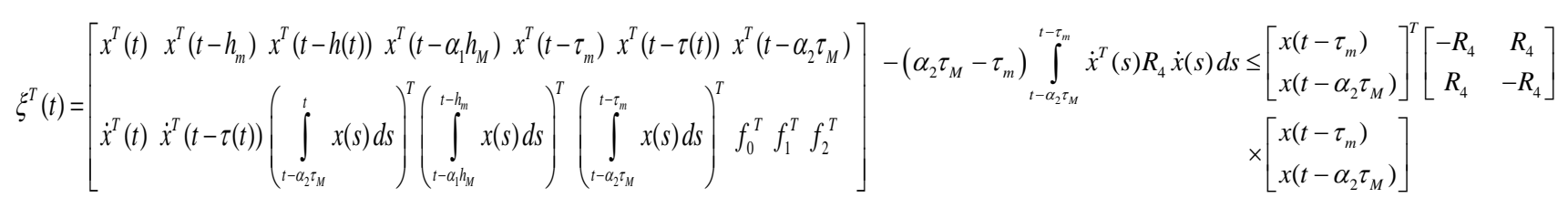

Then, the time derivative of $V(t)$ along the trajectory of system (1) is given by:

where

$$
\dot{V}(t)=\dot{V}_{1}(t)+\dot{V}_{2}(t)+\dot{V}_{3}(t)+\dot{V}_{4}(t)
$$

$$
\begin{aligned}
\dot{V}_{1}(t) & =\dot{x}^{T}(t) P x(t)+x^{T}(t) P \dot{x}(t), \\
\dot{V}_{2}(t) & =x^{T}(t)\left(Q_{1}+Q_{2}+Q_{3}+Q_{4}+Q_{6}+Q_{7}\right) x(t) \\
- & x^{T}\left(t-\alpha_{1} h_{M}\right) Q_{1} x\left(t-\alpha_{1} h_{M}\right)-(1-\dot{h}(t)) x^{T}(t-h(t)) Q_{2} x(t-h(t)) \\
- & x^{T}\left(t-h_{m}\right) Q_{3} x\left(t-h_{m}\right)-(1-\dot{\tau}(t)) x^{T}(t-\tau(t)) Q_{4} x(t-\tau(t)) \\
& -(1-\dot{\tau}(t)) \dot{x}^{T}(t-\tau(t)) Q_{5} \dot{x}(t-\tau(t))+\dot{x}^{T}(t) Q_{5} \dot{x}(t) \\
& -x^{T}\left(t-\alpha_{2} \tau_{M}\right) Q_{6} x\left(t-\alpha_{2} \tau_{M}\right)-x^{T}\left(t-\tau_{m}\right) Q_{7} x\left(t-\tau_{m}\right) \\
\dot{V}_{3}(t) & =\dot{x}^{T}(t)\left(\left(\alpha_{1} h_{M}\right) R_{1}+\left(\alpha_{1} h_{M}-h_{m}\right) R_{2}+\left(\alpha_{2} \tau_{M}\right) R_{3}\right. \\
& \left.+\left(\alpha_{2} \tau_{M}-\tau_{m}\right)^{2} R_{4}\right) \dot{x}(t)-\int_{t-\alpha_{1} h_{M}}^{t} \dot{x}^{T}(s) R_{1} \dot{x}(s) d s \\
& -\int_{t-\alpha_{1} h_{M}}^{t-h_{m}} \dot{x}^{T}(s) R_{2} \dot{x}(s) d s-\int_{t-\alpha_{2} \tau_{M}}^{t} \dot{x}^{T}(s) R_{3} \dot{x}(s) d s \\
& -\left(\alpha_{2} \tau_{M}-\tau_{m}\right) \int_{t-\alpha_{2} \tau_{M}}^{t-\tau_{m}} \dot{x}^{T}(s) R_{4} \dot{x}(s) d s,
\end{aligned}
$$

and:

$$
\begin{aligned}
\dot{V}_{4}(t) & =\dot{x}^{T}(t)\left(\frac{\alpha_{2}^{2} \tau_{M}^{2}}{2} R_{5}+\frac{\left(\alpha_{1}^{2} h_{M}^{2}-h_{m}^{2}\right)}{2} R_{6}\right. \\
& \left.+\frac{\left(\alpha_{2}^{2} \tau_{M}^{2}-\tau_{m}^{2}\right)}{2} R_{7}\right) \dot{x}(t)-\int_{-\alpha_{2} \tau_{M}}^{0} \int_{t+\theta}^{t} \dot{x}^{T}(s) R_{5} \dot{x}(s) d s d \theta \\
& -\int_{-\alpha_{1} h_{M}}^{-h_{m}} \int_{t+\theta}^{t} \dot{x}^{T}(s) R_{6} \dot{x}(s) d s d \theta-\int_{-\tau_{2} \tau_{M}}^{-t} \int_{t+\theta}^{t} \dot{x}^{T}(s) R_{7} \dot{x}(s) d s d \theta .
\end{aligned}
$$

The upper bound of the integral terms in inequality $\dot{V}_{3}(t)$ is estimated as:

$$
\begin{aligned}
& -\int_{t-\alpha_{1} h_{M}}^{t} \dot{x}^{T}(s) R_{1} \dot{x}(s) d s-\int_{t-\alpha_{1} h_{M}}^{t-h_{m}} \dot{x}^{T}(s) R_{2} \dot{x}(s) d s-\int_{t-\alpha_{2} \tau_{M}}^{t} \dot{x}^{T}(s) R_{3} \dot{x}(s) d s \\
& -\left(\alpha_{2} \tau_{M}-\tau_{m}\right) \int_{t-\alpha_{2} \tau_{M}}^{t-\tau_{m}} \dot{x}^{T}(s) R_{4} \dot{x}(s) d s \\
& =-\int_{t-\alpha_{1} h_{M}}^{t-h(t)} \dot{x}^{T}(s) R_{1} \dot{x}(s) d s-\int_{t-h(t)}^{t} \dot{x}^{T}(s) R_{1} \dot{x}(s) d s-\int_{t-\alpha_{\alpha_{1} h_{M}}}^{t-h(t)} \dot{x}^{T}(s) R_{2} \dot{x}(s) d s \\
& -\int_{t-h(t)}^{t-h_{m}} \dot{x}^{T}(s) R_{2} \dot{x}(s) d s-\int_{t-\alpha_{2} \tau_{M}}^{t-\tau(t)} \dot{x}^{T}(s) R_{3} \dot{x}(s) d s-\int_{t-\tau(t)}^{t} \dot{x}^{T}(s) R_{3} \dot{x}(s) d s \\
& -\left(\alpha_{2} \tau_{M}-\tau_{m}\right) \int_{t-\alpha_{2} \tau_{M}}^{t-\tau_{m}} \dot{x}^{T}(s) R_{4} \dot{x}(s) d s
\end{aligned}
$$

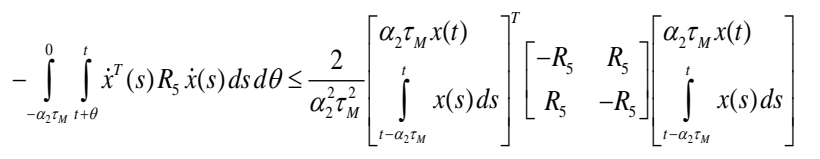

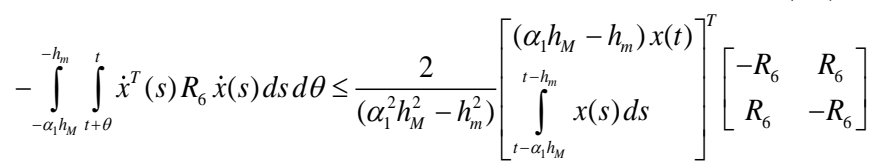$$
\times\left[\begin{array}{c}
\left(\alpha_{1} h_{M}-h_{m}\right) x(t) \\
\int_{t-\alpha_{1} h_{M}}^{t-h_{m}} x(s) d s
\end{array}\right]
$$

$$
\begin{aligned}
-\int_{-\alpha_{2} \tau_{M}}^{-\tau_{m}} \int_{t+\theta}^{t} \dot{x}^{T}(s) R_{7} \dot{x}(s) d s d \theta \leq \frac{2}{\left(\alpha_{2}^{2} \tau_{M}^{2}-\tau_{m}^{2}\right)} & {\left.\left[\int_{t-\alpha_{2} \tau_{M}}^{\left(\alpha_{2} \tau_{M}-\tau_{m}\right) x(t)}\right]^{t-\tau_{m}} x(s) d s\right]^{T}\left[\begin{array}{cc}
-R_{7} & R_{7} \\
R_{7} & -R_{7}
\end{array}\right] } \\
& \times\left[\int_{t-\alpha_{2} \tau_{M}}^{\left(\alpha_{2} \tau_{M}-\tau_{m}\right) x(t)} \begin{array}{l}
t-\tau_{m} \\
\tau_{m}
\end{array}\right] d s
\end{aligned}
$$

By using Lemma 3, an upper bound of integral term of $\dot{V}(t)$ can be obtained as:

$$
\begin{aligned}
-\int_{t-\alpha_{1} h_{M}}^{t-h(t)} \dot{x}^{T}(s)\left(R_{1}+R_{2}\right) \dot{x}(s) d s & \leq\left(\alpha_{1} h_{M}-h(t)\right) \xi^{T}(t) T\left(R_{1}+R_{2}\right)^{-1} T^{T} \xi(t) \\
& +2 \xi^{T}(t) T\left[x(t-h(t))-x\left(t-\alpha_{1} h_{M}\right)\right], \\
-\int_{t-h(t)}^{t} \dot{x}^{T}(s) R_{1} \dot{x}(s) d s & \leq h(t) \xi^{T}(t) Y R_{1}^{-1} Y^{T} \xi(t) \\
+ & 2 \xi^{T}(t) Y[x(t)-x(t-h(t))],
\end{aligned}
$$

$$
\begin{aligned}
-\int_{t-h(t)}^{t-h_{m}} \dot{x}^{T}(s) R_{2} \dot{x}(s) d s & \leq\left(h(t)-h_{m}\right) \xi^{T}(t) W R_{2}^{-1} W^{T} \xi(t) \\
& +2 \xi^{T}(t) W\left[x\left(t-h_{m}\right)-x(t-h(t))\right]
\end{aligned}
$$$$
-\int_{t-\alpha_{2} \tau_{M}}^{t-\tau(t)} \dot{x}^{T}(s) R_{3} \dot{x}(s) d s \leq\left(\alpha_{2} \tau_{M}-\tau(t)\right) \xi^{T}(t) N R_{3}^{-1} N^{T} \xi(t)
$$$$
+2 \xi^{T}(t) N\left[x(t-\tau(t))-x\left(t-\alpha_{2} \tau_{M}\right)\right]
$$

$$
\begin{aligned}
-\int_{t-\tau(t)}^{t} \dot{x}^{T}(s) R_{3} \dot{x}(s) d s & \leq \tau(t) \xi^{T}(t) X R_{3}^{-1} X^{T} \xi(t) \\
& +2 \xi^{T}(t) X[x(t)-x(t-\tau(t))]
\end{aligned}
$$


For any matrices $F_{1}, F_{2}$ with appropriate dimensions, the following equation, from the system (1), verifies:

$2\left[x^{T}(t) F_{1}+\dot{x}^{T}(t) F_{2}\right]\left[A x(t)+A_{1} x(t-h(t))+A_{2} \dot{x}(t-\tau(t))-\dot{x}(t)+f_{0}+f_{1}+f_{2}\right]=0$ (21)

Therefore, combining Equations (10) and (21) yields:

$$
\dot{V}(t) \leq \xi^{T}(t) \Omega \xi(t)
$$

with

$\xi^{T}(t)=\left[\begin{array}{l}x^{T}(t) x^{T}\left(t-h_{m}\right) x^{T}(t-h(t)) x^{T}\left(t-\alpha_{1} h_{M}\right) x^{T}\left(t-\tau_{m}\right) x^{T}(t-\tau(t)) x^{T}\left(t-\alpha_{2} \tau_{M}\right) \\ \dot{x}^{T}(t) \dot{x}^{T}(t-\tau(t))\left(\int_{t-\alpha_{2} \tau_{M}}^{t} x(s) d s\right)^{T}\left(\int_{t-\alpha_{1} h_{M}}^{t-h_{m}} x(s) d s\right)^{T}\left(\int_{t-\alpha_{2} \tau_{M}}^{t-\tau_{m}} x(s) d s\right)^{T} f_{0}^{T} f_{1}^{T} f_{2}^{T}\end{array}\right]$

and $\Omega$ is given in Equation (8).

By using the Schur Complement, it is clear to see that the results $\quad \dot{V}(t)<0$ holds if $\Omega<0, \quad h_{m} \leq h(t) \leq \alpha_{1} h_{M} \quad$ and $\tau_{m} \leq \tau(t) \leq \alpha_{2} \tau_{M}$. Thus, the system (1) is asymptotically stable according to the Lyapunov-Krasovskii theory.

Remark 1: Inspired by the previous works [2-10], some Lyapunov-Krasovskii functional including triple integral terms involving lower and upper bounds of each interval time varying delays have been improved an important role in reduction of conservatism to estimate the maximum allowable delay bound.

Theorem 2. In Case I, if $\alpha_{1} h_{M} \leq h(t) \leq h_{M}\left(0<\alpha_{1}<1\right)$ and $\alpha_{2} \tau_{M} \leq \tau(t) \leq \tau_{M}\left(0<\alpha_{2}<1\right)$, for given positive scalars $h_{m}, h_{M}, \tau_{m}, \tau_{M}, \eta, \mu, \beta_{0}, \beta_{1}$ and $\beta_{2}$, the system (1) with uncertainty (5) and mixed time-varying delays satisfying Equations (2) and (3) is asymptotically stable if there exist symmetric positive definite $n \times n$ matrices $P, Q_{i}(i=1, . ., 7)$,

$R_{j}(j=1, . ., 7)$, for any free matrix variables $T_{a}, Y_{a}, W_{a}, X_{a}$,

$F_{a}(a=1,2)$ and scalars $\varepsilon_{i} \geq 0(i=0,1,2)$ such that the following symmetric LMI holds:

$\left[\begin{array}{ccccc}\Pi & \sqrt{\left(1-\alpha_{1}\right) h_{M}} T & \sqrt{h_{M}} Y & \sqrt{\left(1-\alpha_{1}\right) h_{M}} W & \sqrt{\tau_{M}} X \\ * & -R_{2} & 0 & 0 & 0 \\ * & * & -R_{1} & 0 & 0 \\ * & * & * & -R_{2} & 0 \\ * & * & * & * & -R_{3}\end{array}\right]<0$

where $\Pi=\left(\Pi_{i, j}\right)_{15 \times 15}$

with

$$
\begin{aligned}
& \Pi_{1,1}=Q_{1}+Q_{2}+Q_{3}+Q_{4}+Q_{6}+Q_{7}+Y_{1}+Y_{1}^{T}+X_{1}+X_{1}^{T} \\
& -2 R_{5}-2 R_{6}-2 R_{7}+F_{1} A+A^{T} F_{1}^{T}+\varepsilon_{0} \beta_{0}^{2} \mathrm{I} \\
& \Pi_{1,3}=-Y_{1}+Y_{2}^{T}+F_{1} A_{1} \\
& \Pi_{1,6}=-X_{1}+X_{2}^{T} \\
& \Pi_{1,8}=P-F_{1}+A^{T} F_{2}^{T} \\
& \Pi_{1,9}=F_{1} A_{2} \\
& \Pi_{1,10}=\frac{2}{\tau_{M}} R_{5} \\
& \Pi_{1,11}=\frac{2}{\left(1+\alpha_{1}\right) h_{M}} R_{6} \\
& \Pi_{1,12}=\frac{2}{\left(1+\alpha_{2}\right) \tau_{M}} R_{7} \\
& \Pi_{1,13}=\Omega_{1,14}=\Omega_{1,15}=F_{1} \\
& \Pi_{2,2}=-Q_{1}+W_{1}+W_{1}^{T} \\
& \Pi_{2,3}=-W_{1}+W_{2}^{T} \\
& \Pi_{3,3}=-(1-\mu) Q_{2}+T_{1}+T_{1}^{T}-Y_{2}-Y_{2}^{T}-W_{2}-W_{2}^{T}+\varepsilon_{1} \beta_{1}^{2} \mathrm{I} \\
& \Pi_{3,4}=-T_{1}+T_{2}^{T} \\
& \Pi_{3,8}=A_{1}^{T} F_{2}^{T} \\
& \Pi_{4,4}=-Q_{3}-T_{2}-T_{2}^{T} \\
& \Pi_{5,5}=-R_{4}-Q_{6} \\
& \Pi_{5,7}=R_{4} \\
& \Pi_{6,6}=-(1-\eta) Q_{4}-X_{2}-X_{2}^{T} \\
& \Pi_{7,7}=-R_{4}-Q_{7} \\
& \Pi_{8,8}=Z-F_{2}-F_{2}^{T} \\
& \Pi_{8,9}=F_{2} A_{2} \\
& \Pi_{8,13}=\Omega_{8,14}=\Omega_{8,15}=F_{2} \\
& \Pi_{9,9}=-(1-\eta) Q_{5}+\varepsilon_{2} \beta_{2}^{2} \mathrm{I} \\
& \Pi_{10,10}=-\frac{2}{\tau_{M}^{2}} R_{5} \\
& \Pi_{11,11}=-\frac{2}{\left(1-\alpha_{1}^{2}\right) h_{M}^{2}} R_{6} \\
& \Pi_{12,12}=-\frac{2}{\left(1-\alpha_{2}^{2}\right) \tau_{M}^{2}} R_{7} \\
& \Pi_{13,13}=-\varepsilon_{0} \mathrm{I} \\
& \Pi_{14,14}=-\varepsilon_{1} \mathrm{I} \\
& \Pi_{15,15}=-\varepsilon_{2} \mathrm{I}
\end{aligned}
$$




$$
\begin{aligned}
Z & =Q_{5}+\alpha_{1} h_{M} R_{1}+\left(1-\alpha_{1}\right) h_{M} R_{2}+\alpha_{2} \tau_{M} R_{3}+\left(1-\alpha_{2}\right)^{2} \tau_{M}^{2} R_{4} \\
& +\frac{\tau_{M}^{2}}{2} R_{5}+\frac{\left(1-\alpha_{1}^{2}\right) h_{M}^{2}}{2} R_{6}+\frac{\left(1-\alpha_{2}^{2}\right) \tau_{M}^{2}}{2} R_{7}
\end{aligned}
$$

Proof. Choose a new augmented Lyapunov-Krasovskii functional as:

$$
V(t)=V_{1}(t)+V_{2}(t)+V_{3}(t)+V_{4}(t)
$$

where

$$
\begin{aligned}
V_{1}(t) & =x^{T}(t) P x(t), \\
V_{2}(t) & =\int_{t-\alpha_{1} h_{M}}^{t} x^{T}(s) Q_{1} x(s) d s+\int_{t-h(t)}^{t} x^{T}(s) Q_{2} x(s) d s+\int_{t-h_{M}}^{t} x^{T}(s) Q_{3} x(s) d s \\
& +\int_{t-\tau(t)}^{t} x^{T}(s) Q_{4} x(s) d s+\int_{t-\tau(t)}^{t} \dot{x}^{T}(s) Q_{5} \dot{x}(s) d s+\int_{t-\alpha_{2} \tau_{M}}^{t} x^{T}(s) Q_{6} x(s) d s \\
& +\int_{t-\tau_{M}}^{t} x^{T}(s) Q_{7} x(s) d s, \\
V_{3}(t) & =\int_{-\alpha_{1} h_{M}}^{0} \int_{t+\theta}^{t} \dot{x}^{T}(s) R_{1} \dot{x}(s) d s d \theta+\int_{-h_{M}}^{-\alpha_{1} h_{M}} \int_{t+\theta}^{t} \dot{x}^{T}(s) R_{2} \dot{x}(s) d s d \theta \\
& +\int_{-\alpha_{2} \tau_{M}}^{0} \int_{t+\theta}^{t} \dot{x}^{T}(s) R_{3} \dot{x}(s) d s d \theta+\left(1-\alpha_{2}\right) \tau_{M} \int_{-\alpha_{2} \tau_{M}}^{t} \int_{t+\theta}^{t} \dot{x}^{T}(s) R_{4} \dot{x}(s) d s d \theta \\
V_{4}(t) & =\int_{-\tau_{M}}^{0} \int_{\theta}^{0} \int_{t+\lambda}^{t} \dot{x}^{T}(s) R_{5} \dot{x}(s) d s d \lambda d \theta+\int_{-h_{M}}^{-\alpha_{1} h_{M}} \int_{\theta}^{0} \int_{t+\lambda}^{t} \dot{x}^{T}(s) R_{6} \dot{x}(s) d s d \lambda d \theta \\
& +\int_{-\alpha_{2} \tau_{M}}^{0} \int_{\theta}^{0} \int_{t+\lambda}^{t} \dot{x}^{T}(s) R_{7} \dot{x}(s) d s d \lambda d \theta .
\end{aligned}
$$

with

$$
\zeta^{T}(t)=\left[\begin{array}{l}
x^{T}(t) x^{T}\left(t-\alpha_{1} h_{M}\right) x^{T}(t-h(t)) x^{T}\left(t-h_{M}\right) x^{T}\left(t-\alpha_{2} \tau_{M}\right) x^{T}(t-\tau(t)) x^{T}\left(t-\tau_{M}\right) \\
\dot{x}^{T}(t) \dot{x}^{T}(t-\tau(t))\left(\int_{t-\tau_{M}}^{t} x(s) d s\right)^{T}\left(\int_{t-h_{M}}^{t-\alpha_{h_{M}}} x(s) d s\right)^{T}\left(\int_{t-\tau_{M}}^{t-\alpha_{2} \tau_{M}} x(s) d s\right)^{T} f_{0}^{T} f_{1}^{T} f_{2}^{T}
\end{array}\right]
$$

Then, the time derivative of $V(t)$ along the trajectory of system (1) is given by:

$$
\dot{V}(t)=\dot{V}_{1}(t)+\dot{V}_{2}(t)+\dot{V}_{3}(t)+\dot{V}_{4}(t)
$$

where

$$
\begin{aligned}
& \dot{V}_{1}(t)=\dot{x}^{T}(t) P x(t)+x^{T}(t) P \dot{x}(t), \\
& \dot{V}_{2}(t)=x^{T}(t)\left(Q_{1}+Q_{2}+Q_{3}+Q_{4}+Q_{6}+Q_{7}\right) x(t) \\
& \quad-x^{T}\left(t-\alpha_{1} h_{M}\right) Q_{1} x\left(t-\alpha_{1} h_{M}\right)-(1-\dot{h}(t)) x^{T}(t-h(t)) \\
& \quad Q_{2} x(t-h(t))-x^{T}\left(t-h_{M}\right) Q_{3} x\left(t-h_{M}\right)-(1-\dot{\tau}(t)) x^{T}(t-\tau(t)) \\
& \quad Q_{4} x(t-\tau(t))-(1-\dot{\tau}(t)) \dot{x}^{T}(t-\tau(t)) Q_{5} \dot{x}(t-\tau(t))+\dot{x}^{T}(t) Q_{5} \dot{x}(t) \\
& \quad-x^{T}\left(t-\alpha_{2} \tau_{M}\right) Q_{6} x\left(t-\alpha_{2} \tau_{M}\right)-x^{T}\left(t-\tau_{M}\right) Q_{7} x\left(t-\tau_{M}\right)
\end{aligned}
$$

$$
\begin{gathered}
\dot{V}_{3}(t)=\dot{x}^{T}(t)\left(\left(\alpha_{1} h_{M}\right) R_{1}+\left(h_{M}-\alpha_{1} h_{M}\right) R_{2}+\left(\alpha_{2} \tau_{M}\right) R_{3}\right. \\
\left.+\left(\tau_{M}-\alpha_{2} \tau_{M}\right)^{2} R_{4}\right) \dot{x}(t)-\int_{t-\alpha_{1} h_{M}}^{t} \dot{x}^{T}(s) R_{1} \dot{x}(s) d s \\
-\int_{t-h_{M}}^{t-\alpha_{1} h_{M}} \dot{x}^{T}(s) R_{2} \dot{x}(s) d s-\int_{t-\alpha_{2} \tau_{M}}^{t} \dot{x}^{T}(s) R_{3} \dot{x}(s) d s \\
-\left(1-\alpha_{2}\right) \tau_{M} \int_{t-\alpha_{M} \tau_{M}}^{\dot{x}^{T}}(s) R_{4} \dot{x}(s) d s, \\
\dot{V}_{3}(t) \leq \dot{x}^{T}(t)\left(\left(\alpha_{1} h_{M}\right) R_{1}+\left(h_{M}-\alpha_{1} h_{M}\right) R_{2}+\left(\alpha_{2} \tau_{M}\right) R_{3}\right. \\
\left.+\left(\tau_{M}-\alpha_{2} \tau_{M}\right)^{2} R_{4}\right) \dot{x}(t)-\int_{t-h(t)}^{t} \dot{x}^{T}(s) R_{1} \dot{x}(s) d s \\
\quad-\int_{t-h_{M}}^{t-\alpha_{1} h_{M}} \dot{x}^{T}(s) R_{2} \dot{x}^{t}(s) d s-\int_{t-\tau(t)}^{t} \dot{x}^{T}(s) R_{3} \dot{x}(s) d s \\
-\left(1-\alpha_{2}\right) \tau_{M} \int_{t-\alpha_{M} \tau_{M}} \dot{x}^{T}(s) R_{4} \dot{x}(s) d s
\end{gathered}
$$

and

$$
\begin{aligned}
\dot{V}_{4}(t) & =\dot{x}^{T}(t)\left(\frac{\tau_{M}^{2}}{2} R_{5}+\frac{\left(h_{M}^{2}-\alpha_{1}^{2} h_{M}^{2}\right)}{2} R_{6}+\frac{\left(\tau_{M}^{2}-\alpha_{2}^{2} \tau_{M}^{2}\right)}{2} R_{7}\right) \dot{x}(t) \\
& -\int_{-\tau_{M}}^{0} \int_{t+\theta}^{t} \dot{x}^{T}(s) R_{5} \dot{x}(s) d s d \theta-\int_{-h_{M}}^{-\alpha_{1} h_{M}} \int_{t+\theta}^{t} \dot{x}^{T}(s) R_{6} \dot{x}(s) d s d \theta \\
& -\int_{-\tau_{M}}^{-\alpha_{2} \tau_{M}} \int_{t+\theta}^{t} \dot{x}^{T}(s) R_{7} \dot{x}(s) d s d \theta .
\end{aligned}
$$

The upper bound of the integral terms in inequality $\dot{V}_{3}(t)$ is estimated as:

$$
\begin{aligned}
& -\int_{t-h(t)}^{t} \dot{x}^{T}(s) R_{1} \dot{x}(s) d s-\int_{t-h_{M}}^{t-\alpha_{1} h_{M}} \dot{x}^{T}(s) R_{2} \dot{x}(s) d s-\int_{t-\tau(t)}^{t} \dot{x}^{T}(s) R_{3} \dot{x}(s) d s \\
& -\left(1-\alpha_{2}\right) \tau_{M} \int_{t-\tau_{M}}^{t-\alpha_{2} \tau_{M}} \dot{x}^{T}(s) R_{4} \dot{x}(s) d s \\
& =-\int_{t-h(t)}^{t} \dot{x}^{T}(s) R_{1} \dot{x}(s) d s-\int_{t-h_{M}}^{t-h(t)} \dot{x}^{T}(s) R_{2} \dot{x}(s) d s-\int_{t-h(t)}^{t-\alpha_{1} h_{M}} \dot{x}^{T}(s) R_{2} \dot{x}(s) d s \\
& -\int_{t-\tau(t)}^{t} \dot{x}^{T}(s) R_{3} \dot{x}(s) d s-\left(1-\alpha_{2}\right) \tau_{M} \int_{t-\tau_{M}}^{t-\alpha_{2} \tau_{M}} \dot{x}^{T}(s) R_{4} \dot{x}(s) d s
\end{aligned}
$$

Using Jensen's inequality, such that

$$
\begin{aligned}
& -\left(1-\alpha_{2}\right) \tau_{M} \int_{t-\tau_{M}}^{t-\alpha_{2} \tau_{M}} \dot{x}^{T}(s) R_{4} \dot{x}(s) d s \leq\left[\begin{array}{l}
x\left(t-\alpha_{2} \tau_{M}\right) \\
x\left(t-\tau_{M}\right)
\end{array}\right]^{T}\left[\begin{array}{cc}
-R_{4} & R_{4} \\
R_{4} & -R_{4}
\end{array}\right]\left[\begin{array}{l}
x\left(t-\alpha_{2} \tau_{M}\right) \\
x\left(t-\tau_{M}\right)
\end{array}\right] \\
& -\int_{-\tau_{M}}^{0} \int_{t+\theta}^{t} \dot{x}^{T}(s) R_{5} \dot{x}(s) d s d \theta \leq \frac{2}{\tau_{M}^{2}}\left[\begin{array}{c}
\tau_{M} x(t) \\
\int_{t-\tau_{M}}^{t} x(s) d s
\end{array}\right]^{T}\left[\begin{array}{cc}
-R_{5} & R_{5} \\
R_{5} & -R_{5}
\end{array}\right]\left[\begin{array}{l}
\tau_{M} x(t) \\
\int_{t-\tau_{M}}^{t} x(s) d s
\end{array}\right]
\end{aligned}
$$


$\left.\left.-\int_{-h_{M}}^{-\alpha_{1} h_{M}} \int_{t+\theta}^{t} \dot{x}^{T}(s) R_{6} \dot{x}(s) d s d \theta \leq \frac{2}{\left(h_{M}^{2}-\alpha_{1}^{2} h_{M}^{2}\right)}\left[\int_{t-h_{M}}^{\left(1-\alpha_{1}\right) h_{M} x(t)}\right]^{t-\alpha_{\alpha} h_{M}}\right]^{T} x(s) d s\right]\left[\begin{array}{cc}-R_{6} & R_{6} \\ R_{6} & -R_{6}\end{array}\right]\left[\int_{t-h_{M}}^{\left(1-\alpha_{1}\right) h_{M} x(t)} x(s) d s\right]$

$-\int_{-\tau_{M}}^{-\alpha_{2} \tau_{M}} \int_{t+\theta}^{t} \dot{x}^{T}(s) R_{7} \dot{x}(s) d s d \theta \leq \frac{2}{\left(\tau_{M}^{2}-\alpha_{2}^{2} \tau_{M}^{2}\right)}\left[\begin{array}{c}\left(1-\alpha_{2}\right) \tau_{M} x(t) \\ t-\alpha_{2} \tau_{M} \\ \int_{t-\tau_{M}}^{T} x(s) d s\end{array}\right]^{T}\left[\begin{array}{cc}-R_{7} & R_{7} \\ R_{7} & -R_{7}\end{array}\right]\left[\int_{t-\tau_{M}}^{\left(1-\alpha_{2}\right) \tau_{M} x(t)} x(s) d s\right]$

(30)

By using Lemma 3, an upper bound of integral term of $\dot{V}(t)$ can be obtained as:

$$
\begin{aligned}
-\int_{t-h_{M}}^{t-h(t)} \dot{x}^{T}(s) R_{2} \dot{x}(s) d s & \leq\left(h_{M}-h(t)\right) \zeta^{T}(t) T R_{2}^{-1} T^{T} \zeta(t) \\
+ & 2 \zeta^{T}(t) T\left[x(t-h(t))-x\left(t-h_{M}\right)\right] \\
-\int_{t-h(t)}^{t} \dot{x}^{T}(s) R_{1} \dot{x}(s) d s & \leq h(t) \zeta^{T}(t) Y R_{1}^{-1} Y^{T} \zeta(t) \\
+ & 2 \zeta^{T}(t) Y[x(t)-x(t-h(t))] \\
-\int_{t-h(t)}^{t-\alpha_{1} h_{M}} \dot{x}^{T}(s) R_{2} \dot{x}(s) d s & \leq\left(h(t)-\alpha_{1} h_{M}\right) \zeta^{T}(t) W R_{2}^{-1} W^{T} \zeta(t) \\
& +2 \zeta^{T}(t) W\left[x\left(t-\alpha_{1} h_{M}\right)-x(t-h(t))\right] \\
-\int_{t-\tau(t)}^{t} \dot{x}^{T}(s) R_{3} \dot{x}(s) d s & \leq \tau(t) \zeta^{T}(t) X R_{3}^{-1} X^{T} \zeta(t) \\
& +2 \zeta^{T}(t) X[x(t)-x(t-\tau(t))]
\end{aligned}
$$

From Equation (5), the following inequalities hold:

$$
\left\{\begin{array}{l}
\beta_{0}^{2} x^{T}(t) x(t)-f_{0}^{T} f_{0} \geq 0 \\
\beta_{1}^{2} x^{T}\left(t-\tau_{1}(t)\right) x\left(t-\tau_{1}(t)\right)-f_{1}^{T} f_{1} \geq 0 \\
\beta_{2}^{2} \dot{x}^{T}\left(t-\tau_{2}(t)\right) \dot{x}\left(t-\tau_{2}(t)\right)-f_{2}^{T} f_{2} \geq 0
\end{array}\right.
$$

Further, for any scalars $\varepsilon_{i}>0(i=0,1,2)$, it follows from Equation (35), that

$$
\left\{\begin{array}{l}
\varepsilon_{0}\left[\beta_{0}^{2} x^{T}(t) x(t)-f_{0}^{T} f_{0}\right] \geq 0, \\
\varepsilon_{1}\left[\beta_{1}^{2} x^{T}\left(t-\tau_{1}(t)\right) x\left(t-\tau_{1}(t)\right)-f_{1}^{T} f_{1}\right] \geq 0, \\
\varepsilon_{2}\left[\beta_{2}^{2} \dot{x}^{T}\left(t-\tau_{2}(t)\right) \dot{x}\left(t-\tau_{2}(t)\right)-f_{2}^{T} f_{2}\right] \geq 0,
\end{array}\right.
$$

Therefore, combining Equations (25) and (36) yields:

$$
\dot{V}(t) \leq \zeta^{T}(t) \Pi \zeta(t),
$$

with

$$
\zeta^{T}(t)=\left[\begin{array}{l}
x^{T}(t) x^{T}\left(t-\alpha_{1} h_{M}\right) x^{T}(t-h(t)) x^{T}\left(t-h_{M}\right) \\
x^{T}\left(t-\alpha_{2} \tau_{M}\right) x^{T}(t-\tau(t)) x^{T}\left(t-\tau_{M}\right) \\
\dot{x}^{T}(t) \dot{x}^{T}(t-\tau(t))\left(\int_{t-\tau_{M}}^{t} x(s) d s\right)^{T}\left(\int_{t-h_{M}}^{t-\alpha_{1} h_{M}} x(s) d s\right)^{T} \\
\left(\int_{t-\tau_{M}}^{t-\alpha_{2} \tau_{M}} x(s) d s\right)^{T} f_{0}^{T} f_{1}^{T} f_{2}^{T}
\end{array}\right]
$$

and $\Pi$ is given in Equation (23). By using the Schur Complement, it is clear to see that the results $\dot{V}(t)<0$ holds if $\Pi<0, \alpha_{1} h_{M} \leq h(t) \leq h_{M}$, and $\alpha_{2} \tau_{M} \leq \tau(t) \leq \tau_{M}$.

Thus, the system (1) is asymptotically stable according to the Lyapunov-Krasovskii method.

Theorem 3. In Case II, for given positive scalars $h_{m}, h_{M}$,

$\tau_{m}, \tau_{M}, \eta, \mu, \beta_{0}, \beta_{1}$ and $\beta_{2}$, the system (1) with uncertainty (5) and mixed time-varying delays satisfying Equations (2) and (3) is asymptotically stable if there exist symmetric positive definite $n \times n$ matrices $P, Q_{i}(i=1,3, . .7), R_{j}(j=1, . ., 7)$, for any free matrix variables $T_{a}, Y_{a}, W_{a}, N_{a}, X_{a}, F_{a}(a=1,2)$ and scalars $\varepsilon_{i} \geq 0(i=0,1,2)$ such that the following symmetric LMI holds:

$$
\begin{aligned}
& {\left[\begin{array}{cccccc}
\Sigma & \sqrt{\alpha_{1} h_{M}-h_{m}} T & \sqrt{\alpha_{1} h_{M}} Y & \sqrt{\alpha_{1} h_{M}-h_{m}} W & \sqrt{\alpha_{2} \tau_{M}-\tau_{m}} N & \sqrt{\alpha_{2} \tau_{M}} X \\
* & -\left(R_{1}+R_{2}\right) & 0 & 0 & 0 & 0 \\
* & * & -R_{1} & 0 & 0 & 0 \\
* & * & * & -R_{2} & 0 & 0 \\
* & * & * & * & -R_{3} & 0 \\
* & * & * & * & * & -R_{3}
\end{array}\right]} \\
& \text { where } \quad \Sigma=\left(\Sigma_{i, j}\right)_{15 \times 15}
\end{aligned}
$$

with

$$
\begin{aligned}
\Sigma_{1,1} & =Q_{1}+Q_{3}+Q_{4}+Q_{6}+Q_{7}+Y_{1}+Y_{1}^{T}+X_{1}+X_{1}^{T} \\
& -2 R_{5}-2 R_{6}-2 R_{7}+F_{1} A+A^{T} F_{1}^{T}+\varepsilon_{0} \beta_{0}^{2} \mathrm{I} \\
\Sigma_{1,3} & =-Y_{1}+Y_{2}^{T}+F_{1} A_{1} \\
\Sigma_{1,6} & =-X_{1}+X_{2}^{T} \\
\Sigma_{1,8} & =P-F_{1}+A^{T} F_{2}^{T} \\
\Sigma_{1,9} & =F_{1} A_{2} \\
\Sigma_{1,10} & =\frac{2}{\alpha_{2} \tau_{M}} \\
\Sigma_{1,11} & =\frac{2}{\left(\alpha_{1} h_{M}+h_{m}\right)} R_{6} \\
\Sigma_{1,12} & =\frac{2}{\left(\alpha_{2} \tau_{M}+\tau_{m}\right)} R_{7} \\
\Sigma_{1,13} & =\Omega_{1,14}=\Omega_{1,15}=F_{1} \\
\Sigma_{2,2} & =-Q_{3}+W_{1}+W_{1}^{T} \\
\Sigma_{2,3} & =-W_{1}+W_{2}^{T} \\
\Sigma_{3,3} & =T_{1}+T_{1}^{T}-Y_{2}-Y_{2}^{T}-W_{2}-W_{2}^{T}+\varepsilon_{1} \beta_{1}^{2} \mathrm{I} \\
\Sigma_{3,4}= & -T_{1}+T_{2}^{T} \\
\Sigma_{3,8}= & A_{1}^{T} F_{2}^{T} \\
\Sigma_{4,4}= & -Q_{1}-T_{2}-T_{2}^{T} \\
\Sigma_{5,5} & =-R_{4}-Q_{7} \\
&
\end{aligned}
$$


$\Sigma_{5,7}=R_{4}$

$\Sigma_{6,6}=-(1-\eta) Q_{4}+N_{1}+N_{1}^{T}-X_{2}-X_{2}^{T}$

$\Sigma_{7,7}=-R_{4}-Q_{6}-N_{2}-N_{2}^{T}$

$\Sigma_{8,8}=M-F_{2}-F_{2}^{T}$

$\Sigma_{8,9}=F_{2} A_{2}$

$\Sigma_{8,13}=\Omega_{8,14}=\Omega_{8,15}=F_{2}$

$\Sigma_{9,9}=-(1-\eta) Q_{5}+\varepsilon_{2} \beta_{2}^{2} \mathrm{I}$

$\Sigma_{10,10}=-\frac{2}{\alpha_{2}^{2} \tau_{M}^{2}} R_{5}$

$\Sigma_{11,11}=-\frac{2}{\left(\alpha_{1}^{2} h_{M}^{2}-h_{m}^{2}\right)} R_{6}$

$\Sigma_{12,12}=-\frac{2}{\left(\alpha_{2}^{2} \tau_{M}^{2}-\tau_{m}^{2}\right)} R_{7}$

$\Sigma_{13,13}=-\varepsilon_{0} \mathrm{I}$

$\Sigma_{14,14}=-\varepsilon_{1} \mathrm{I}$

$\Sigma_{15,15}=-\varepsilon_{2} \mathrm{I}$

$\xi^{T}(t)=\left[\begin{array}{l}x^{T}(t) x^{T}\left(t-h_{m}\right) x^{T}(t-h(t)) x^{T}\left(t-\alpha_{1} h_{M}\right) x^{T}\left(t-\tau_{m}\right) x^{T}(t-\tau(t)) x^{T}\left(t-\alpha_{2} \tau_{M}\right) \\ \dot{x}^{T}(t) \dot{x}^{T}(t-\tau(t))\left(\int_{t-\alpha_{2} \tau_{M}}^{t} x(s) d s\right)^{T}\left(\int_{t-\alpha_{1} h_{M}}^{t-h_{m}} x(s) d s\right)^{T}\left(\int_{t-\alpha_{2} \tau_{M}}^{t-\tau_{m}} x(s) d s\right)^{T} f_{0}^{T} f_{1}^{T} f_{2}^{T}\end{array}\right]$

In Case II, a Lyapunov-Krasovskii functional can be chosen as (8) with $Q_{2}=0$. Similar to the above analysis, one can get that the results $\dot{V}(t)<0$ holds if $\Sigma<0$, $h_{m} \leq h(t) \leq \alpha_{1} h_{M}$, and $\tau_{m} \leq \tau(t) \leq \alpha_{2} \tau_{M}$.Thus, the proof is completed.

Remark 2: By introducing a new class of augmented Lyapunov-Krasovskii functional approach, new delaydecomposition stability criteria for nonlinear neutral systems with mixed time-varying delays are obtained in Theorems 1-3. The proposed augmented Lyapunov functional using the novel triple integral inequality is more robust than existing results in literature. It gives the upper bounds of time-varying delays $h(t), \tau(t)$ for the asymptotic stability of system (1) which can be provided larger stability domain. In addition, by applying free-weighting matrices and Jensen integral inequality, our decomposition approach, developed in Theorems 1-3, yields a much less conservative delay bounds and extends the feasible region of stability method for system (1).

Remark 3: In order to derive a fewer restrictive stability criteria for system (1), many free-weighting matrix variables are employed in Theorems 1-3. In fact, this technique of decision variables reduces the computational complexity of the obtained stability approach which is less than the previous methods.

Remark 4: In this work and from the practical point of view, several problems related to this studied field are still open such as singular descriptor systems with multiple mixed time-varying delays, chaotic systems with varying delays and neural networks systems.

\section{ILLUSTRATIVE EXAMPLES}

In this section, two examples are presented in order to show the less conservatism of the elaborated stability condition and to demonstrate the effectiveness of the proposed approach.

\section{Example 1.}

Consider the following nonlinear neutral system with mixed time-varying delays, as given in [10]:

$$
A=\left[\begin{array}{cc}
-1.2 & 0.1 \\
-0.1 & -1
\end{array}\right], A_{1}=\left[\begin{array}{cc}
-0.6 & 0.7 \\
-1 & -0.8
\end{array}\right], A_{2}=\left[\begin{array}{ll}
c & 0 \\
0 & c
\end{array}\right],
$$

where $\mathrm{O} \leq|c|<1, \quad \beta_{0} \geq 0, \beta_{1} \geq 0$, and $\beta_{2} \geq 0$.

Case I. For $c=0.1, \quad \beta_{1}=0.1, \tau_{M}=1, \mu=0.5, \eta=0$, $\alpha_{2}=0.2$ and different values of $\beta_{2}$, the maximal allowable delay of $h_{M}$ estimated by Theorems 1 and 2 are illustrated in Table 1. This table shows the numerical results for different values of $\beta_{2}, \beta_{0}=0$ and $\beta_{0}=0.1$. As $\beta_{2}$ increases, $h_{M}$ decreases. In addition, the proposed stability technique gives a much less conservative result than other recent ones.

Case II. For $\beta_{0}=0.1, \beta_{1}=0.2, \beta_{2}=0.1, \alpha_{2}=0.2, \mu=1$ and different values of $c$, the maximum admissible upper bound on the allowable time delay of $h_{M}=\tau_{M}$ obtained from Theorem 1 are listed in Table 2. As $c$ increases, $h_{M}$ decreases. It is clear that the proposed stability method in this paper provides larger upper bounds of delay system than the previous results for different values of $c$.

Case III. For $\mathrm{c}=0.1, \alpha_{2}=0.2, \beta_{1}=0.1, \beta_{2}=0, \beta_{0}=0$ and $\beta_{0}=0.1$, and different values of $\mu=\eta$, the maximum upper bounds on the allowable delay of $h_{M}=\tau_{M}$ obtained from Theorems 1 and 2 are illustrated in Table 3. As ${ }^{\mu}$ increases, $h_{M}$ decreases. The presented stability criterion is less conservative than existing results.

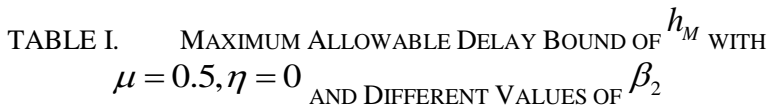

\begin{tabular}{lcccc}
\hline$\beta_{0}=0$ & & & & \\
\hline$\beta_{2}$ & 0 & 0.1 & 0.2 & 0.3 \\
\hline Rakkiyappan et al.[18] & 1.4886 & 1.2437 & 0.9921 & 0.7367 \\
& & & & \\
Lakshmanan et al.[13] & 1.6325 & 1.3386 & 1.0816 & 0.8563 \\
& & & & \\
Cheng et al. [23] & 1.6865 & 1.3721 & 1.0923 & 0.8613
\end{tabular}




\begin{tabular}{lcccc}
\hline Qiu and Zhang [21] & 2.2937 & 1.8505 & 1.4565 & 1.1105 \\
& & & & \\
Theorem1 $\left(\alpha_{1}=0.25\right)$ & 6.0782 & 5.1772 & 3.4872 & 1.9325 \\
Theorem2 $\left(\alpha_{1}=0.1\right)$ & 4.7856 & 4.0752 & 2.7424 & 1.5156 \\
\hline$\beta_{0}=0.1$ & & & & \\
\hline$\beta_{2}$ & 0 & 0.1 & 0.2 & 0.3 \\
\hline Rakkiyappan et al.[18] & 1.3244 & 1.0901 & 0.8475 & 0.6300 \\
Lakshmanan et al.[13] & 1.4440 & 1.1950 & 0.9734 & 0.7760 \\
Cheng et al. [23] & 1.4721 & 1.2466 & 0.9996 & 0.7804 \\
Qiu and Zhang [21] & 2.0417 & 1.6541 & 1.3062 & 0.9982 \\
Theorem1 $\left(\alpha_{1}=0.25\right)$ & 5.6888 & 4.9444 & 3.4125 & 1.9128 \\
Theorem2 $\left(\alpha_{1}=0.1\right)$ & 4.4785 & 3.8915 & 2.6834 & 1.5000 \\
\hline
\end{tabular}

TABLE II. MAXIMUM UPPER BOUND OF $h_{M}=\tau_{M}$ WITH DIFFERENT VALUES OF $C$

\begin{tabular}{lccc}
\hline$c$ & 0.1 & 0.2 & 0.3 \\
\hline Zhang and Yu [17] & 0.4911 & 0.4125 & 0.3382 \\
& & & \\
Qiu et al. [15] & 1.8567 & 1.6242 & 1.3917 \\
& & & \\
Qiu and Zhang [21] & 2.1916 & 1.6632 & 1.4743 \\
& & & \\
Theorem1 $\left(\alpha_{1}=0.1\right)$ & 6.4209 & 5.5817 & 4.7240 \\
\hline$c$ & 0.4 & 0.5 & 0.6 \\
\hline Zhang and Yu [17] & 0.2671 & 0.1975 & 0.1294 \\
Qiu et al. [15] & 1.1592 & 0.9270 & 0.6945 \\
Qiu and Zhang [21] & 1.2396 & 0.9288 & 0.7446 \\
Theorem1 $\left(\alpha_{1}=0.1\right)$ & 3.8303 & 2.8351 & 1.5622 \\
\hline
\end{tabular}

TABLE III. MAXIMUM UPPER BOUND OF $h_{M}=\tau_{M}$ WITH DIFFERENT VALUES OF $\mu=\eta$

\begin{tabular}{|c|c|c|c|c|}
\hline & $\beta_{0}=0$ & $\beta_{1}=0.1$ & \multicolumn{2}{|c|}{$\beta_{0}=0.1 \quad \beta_{1}=0.1$} \\
\hline$\mu=\eta$ & 0 & 0.5 & 0 & 0.5 \\
\hline Chen et al. [23] & 2.7423 & 1.1425 & 1.8753 & 1.0097 \\
\hline Liu [22] & 2.7429 & 1.4462 & 1.8895 & 1.1485 \\
\hline Qiu and Zhang [21] & 3.8066 & 1.6402 & 2.6039 & 1.4534 \\
\hline Theorem1 $\left(\alpha_{1}=0.6\right)$ & 8.1497 & 2.3438 & 5.6008 & 2.1964 \\
\hline Theorem $2\left(\alpha_{1}=0.7\right)$ & 5.8444 & 1.6738 & 4.0165 & 1.5683 \\
\hline
\end{tabular}

\section{Example 2.}

Consider the mixed time-varying delay systems as depicted in Equation (40):

$$
\begin{aligned}
& A=\left[\begin{array}{cc}
-2 & 0.5 \\
0 & -1
\end{array}\right], A_{1}=\left[\begin{array}{cc}
1 & 0.4 \\
0.4 & -1
\end{array}\right], A_{2}=\left[\begin{array}{cc}
0.2 & 1 \\
0 & 0.2
\end{array}\right], \\
& \text { with }
\end{aligned}
$$

$$
\begin{aligned}
& f_{0}^{T}(t, x(t)) f_{0}(t, x(t)) \leq \beta_{0}^{2} x^{T}(t) x(t) \text { and } \\
& f_{1}^{T}(t, x(t-h(t))) f_{1}(t, x(t-h(t))) \leq \beta_{1}^{2} x^{T}(t-h(t)) x(t-h(t)) .
\end{aligned}
$$

While using the parameters $\alpha_{1}=0.1, \alpha_{2}=0.2, \eta=\mu=0$, $\beta_{0}=0.2, \beta_{1}=0.1$ and $\beta_{2}=0$, the upper bound of Time Delay $h_{M}=\tau_{M}$ obtained from Theorem 3 is feasible for any delay $0<h_{M} \leq 3.8561$.

It is remarkable that this proposed criterion is much less conservative than the results shown in $[14,16]$.

\section{CONCLUSION}

This paper studied the problem of asymptotic stability for nonlinear neutral mixed time-varying delays systems. By using the Lyapunov-Krasovskii functional with triple integral terms and free weighting matrices approach, new delaydependent stability criteria are derived by developing a delay decomposition technique. The elaborated approach is then expressed in terms of LMIs. Finally, numerical simulations have been investigated in order to show the robustness and the flexibility of the proposed stability method.

\section{REFERENCES}

[1] M.N. Alpaslan Parlakçı, Delay-Dependent Robust Stability criteria for uncertain neutral systems with mixed time-varying discrete and neutral delays, Asian Journal of Control, vol. 9, no. 4, pp. 411-421, December 2007

[2] I. Amri, D. Soudani and M. Benrejeb, A Delay Decomposition Approach for Exponential Stability of Perturbed Neutral Systems with Mixed Delay, $7^{\text {th }}$ International Multi-Conference on Systems, Signals and Devices (SSD'10), Amman, Jordan, 2010.

[3] P. L. Liu, A delay decomposition approach to stability analysis of neutral systems with time varying delay, Appl. Math. Modell, vol. 37, pp. 5013-5026, 2013

[4] F. Qiu, B.Cui, and Y. Ji, A delay-dividing approach to stability of neutral system with mixed delays and nonlinear perturbations, Applied Mathematical Modelling, vol. 34, pp.3701-3707, 2010.

[5] Ali, MS, On exponential stability of neutral delay differential system with nonlinear uncertainties, Nonlinear Sci. Numer. Simul, vol. 17, pp. 2595-2601, 2012.

[6] J. Cheng, H. Zhu, S. M. Zhong, G. H. Li, Novel delay-dependent robust stability criteria for neutral systems with mixed time-varying delays and nonlinear perturbations, Appl. Math. Comput, vol. 219, pp. 7741-7753, 2013.

[7] Junjun HUI, Hexin ZHANG, Xiangyu KONG, and Fei MENG, A Less Conservative Robust Stability Criteria for Neutral System with Mixed Time-varying Delays and Nonlinear Perturbations, Journal of Computational Information Systems, vol. 4, no. 10, pp. 1543-1553, 2014.

[8] X.M. Zhang, Q.L. Han, A delay decomposition approach to delaydependent stability for linear systems with time-varying delays, International Journal of Robust and Nonlinear Control, vol. 19, no. 17, pp. 1922-1930, 2009.

[9] S. Boyd, L. EL Ghaoui, E. Feron, V. Balakrishnan, Linear matrix inequalities in system and control theory, Studies in Applied Mathematics, SIAM, Philadelphia, USA, 1994.

[10] O.M. Kwon, J.H. Park, S.M. Lee, An improved delay-dependent criterion for asymptotic stability of uncertain dynamic systems with time-varying delays, J. Optim. Theory Appl., vol. 145, pp. 343-353, 2010

[11] F. Gouaisbaut and D. Peaucelle, Delay-dependent stability of time delay systems, 5th IFAC Symposium on Robust Control (ROCOND'06), Toulouse, France, 5-7 July 2006.

[12] Q. L. Han, Robust stability for a class of linear systems with timevarying delay and nonlinear perturbations, Computer and Mathematics with Applications, vol.47, pp.1201-1209, 2004. 
[13] S. Lakshmanan, T. Senthilkumar, and P. Balasubramaniam, Improved results on robust stability of neutral systems with mixed time-varying delays and nonlinear perturbations, Applied Mathematical Modelling, vol. 35, pp. 5355-5368, 2011.

[14] J.H. Park, Novel robust stability criterion for a class of neutral systems with mixed delays and nonlinear perturbations, Applied Mathematics and Computation, vol. 161, no. 2, pp. 413-421, 2005.

[15] F. Qiu, B. Cui, and Y. Ji, Further results on robust stability of neutral system with mixed time-varying delays and nonlinear perturbations, Nonlinear Analysis: Real World Applications, vol. 11, pp. 895-906, 2010.

[16] L. L. Xiong, S. M. Zhong, and D. Y. Li, Novel delay-dependent asymptotical stability of neutral systems with nonlinear perturbations, Journal of Computational and Applied Mathematics, vol. 232, pp. 505513, 2009.

[17] [17] W. A. Zhang and L.Yu, Delay-dependent robust stability of neutral systems with mixed delays and nonlinear perturbations, Acta Automatica Sinica, vol. 33, pp. 863-866, 2007.

[18] [18] R. Rakkiyappan, P. Balasubramaniam, R. Krishnasamy, Delay dependent stability analysis of neutral systems with mixed time-varying delays and nonlinear perturbations, J. Comput. Appl. Math., vol. 235, pp. 2147-2156, 2011.

[19] I. Amri and D. Soudani, Robust Exponential Stability of Uncertain Perturbed Systems with TimeVarying Delays, IFAC-12th LSS symposium: Theory and Applications, Lille, France, 2010.

[20] I. Amri, D. Soudani and M. Benrejeb, On Robust $\alpha$-Stability Analysis of Uncertain Neutral Systems with Time Varying Delays: A Novel Augmented Lyapunov Functional Approach, 8th International MultiConference on Systems, Signals and Devices (SSD’11), Sousse, Tunisia, 2011.

[21] F. Qiu and Q. Zhang, Robust Stability of Neutral System with Mixed Time-Varying Delays and nonlinear perturbations Using Delay Decomposition Approach, Abstract and Applied Analysis, vol. 2014, Article ID 825715, 11 pages, 2014.

[22] P. L. Liu, Robust stability for neutral time-varying delay systems with non-linear perturbations, International Journal of Innovative Computing, Information and Control, vol.7, no. 10, pp.5749-5760, 2011.

[23] Y. Chen, A. K. Xue, R. Q. Lu, and S. S. Zhou, On robust exponential stability of uncertain neutral systems with time-varying delays and nonlinear perturbations, Nonlinear Analysis, vol. 68, pp.2464-2470, 2008. 